PROCEEDINGS OF THE

AMERICAN MATHEMATICAL SOCIETY

Volume 137, Number 1, January 2009, Pages 19-26

S 0002-9939(08)09437-9

Article electronically published on July 29, 2008

\title{
PARAMETRIC DECOMPOSITION OF POWERS OF PARAMETER IDEALS AND SEQUENTIALLY COHEN-MACAULAY MODULES
}

\author{
NGUYEN TU CUONG AND HOANG LE TRUONG
}

(Communicated by Bernd Ulrich)

\begin{abstract}
Let $M$ be a finitely generated module of dimension $d$ over a Noetherian local ring $(R, \mathfrak{m})$ and $\mathfrak{q}$ an ideal generated by a system of parameters $\underline{x}=\left(x_{1}, \ldots, x_{d}\right)$ of $M$. For each positive integer $n$, set

$$
\Lambda_{d, n}=\left\{\alpha=\left(\alpha_{1}, \ldots, \alpha_{d}\right) \in \mathbb{Z}^{d} \mid \alpha_{i} \geqslant 1,1 \leqslant i \leqslant d \text { and } \sum_{i=1}^{d} \alpha_{i}=d+n-1\right\}
$$

and $\mathfrak{q}(\alpha)=\left(x_{1}^{\alpha_{1}}, \ldots, x_{d}^{\alpha_{d}}\right)$ for each $\alpha \in \Lambda_{d, n}$. Then we prove in this note that $M$ is a sequentially Cohen-Macaulay module if and only if there exists a good system of parameters $\underline{x}$ such that the equality $\mathfrak{q}^{n} M=\bigcap_{\alpha \in \Lambda_{d, n}} \mathfrak{q}(\alpha) M$ holds true for all $n \geq 1$. As an application, we show that the sequentially CohenMacaulayness of a module can be characterized by a very special expression of the Hilbert-Samuel polynomial of a good parameter ideal.
\end{abstract}

\section{INTRODUCTION}

Throughout this paper we denote by $R$ a commutative Noetherian local ring with the maximal ideal $\mathfrak{m}$ and by $M$ a finitely generated $R$-module with $\operatorname{dim} M=d$. Let $\underline{x}=x_{1}, \ldots, x_{d}$ be a system of parameters of the module $M$ and $\mathfrak{q}=\left(x_{1}, \ldots, x_{d}\right)$ the parameter ideal of $M$ generated by $\underline{x}$. For each integer $n \geqslant 1$, we set

$$
\Lambda_{d, n}=\left\{\left(\alpha_{1}, \ldots, \alpha_{d}\right) \in \mathbb{Z}^{d} \mid \alpha_{i} \geqslant 1 \text { for all } 1 \leqslant i \leqslant d \text { and } \sum_{i=1}^{d} \alpha_{i}=d+n-1\right\} .
$$

Let $\mathfrak{q}(\alpha)=\left(x_{1}^{\alpha_{1}}, \ldots, x_{d}^{\alpha_{d}}\right)$ for each $\alpha=\left(\alpha_{1}, \ldots, \alpha_{d}\right) \in \Lambda_{d, n}$. We say that the system $\underline{x}$ of parameters has the property of parametric decomposition if the equality $\mathfrak{q}^{n} M=$ $\bigcap \mathfrak{q}(\alpha) M$ holds true for all $n \geqslant 1$. The main purpose of this paper is to study the $\alpha \in \Lambda_{d, n}$

question of when a given system of parameters of $M$ has the property of parametric decomposition. Notice that Heinzer, Ratliff and Shah [HRS, Theorem 2.4] proved that an $R$-regular sequence always has the property of parametric decomposition. Later, Goto and Shimoda [GS1, Theorem 1.1] showed that the converse is also true

Received by the editors November 15, 2006, and, in revised form, September 11, 2007, and November 18, 2007.

2000 Mathematics Subject Classification. Primary 13H10; Secondary 13 H99.

Key words and phrases. Parametric decomposition, sequentially Cohen-Macaulay module, dimension filtration, good system of parameters.

(C)2008 American Mathematical Society Reverts to public domain 28 years from publication 
when each element of the sequence is a non-zerodivisor in $R$. Moreover, they gave in [GS2, Theorem 1.1] a characterization of $R$ with $\operatorname{dim} R \geqslant 2$, in which every system of parameters of $R$ has the property of parametric decomposition. In order to generalize this result of Goto and Shimoda, let us recall some notions which were defined in $\left[\mathrm{CC}\right.$. A filtration $\mathcal{D}: H_{\mathfrak{m}}^{0}(M)=D_{0} \subset D_{1} \subset \ldots \subset D_{t}=M$ of submodules of $M$ is said to be a dimension filtration if $D_{i-1}$ is the largest submodule of $D_{i}$ with $\operatorname{dim} D_{i-1}<\operatorname{dim} D_{i}$ for all $i=t, t-1, \ldots, 1$. If $D_{i} / D_{i-1}$ is CohenMacaulay for all $1 \leq i \leq t, M$ is called a sequentially Cohen-Macaulay module. A system $\underline{x}=x_{1}, \ldots, x_{d}$ of parameters of $M$ is called a good system of parameters of $M$ if $D_{i} \cap\left(x_{d_{i}+1}, \ldots, x_{d}\right) M=0$ for all $0 \leq i \leq t-1$, where $d_{i}=\operatorname{dim} D_{i}$. Now, we restrict our interest in the above question to the set of all good systems of parameters of $M$. It turns out that the property of parametric decomposition of a good system of parameters can be characterized by the sequentially CohenMacaulayness of the module. The following theorem is the main result of this paper.

Theorem 1.1. The following statements are equivalent:

(i) $M$ is a sequentially Cohen-Macaulay module.

(ii) Every good system of parameters of $M$ has the property of parametric decomposition.

(iii) There exists a good system of parameters of $M$ having the property of parametric decomposition.

As a consequence of Theorem 1.1 we again obtain the main result of GotoShimoda [GS2, Theorem 1.1]. It should be noted here that Theorem 1.1 of GS2 was stated for local rings, but its proof still works in the module case.

Corollary 1.2. Let $\operatorname{dim} M \geqslant 2$ and $H_{\mathfrak{m}}^{0}(M)$ the $0^{\text {th }}$ local cohomology module of $M$ with respect to the maximal ideal $\mathfrak{m}$. Then the following statements are equivalent:

(i) $M / H_{\mathfrak{m}}^{0}(M)$ is a Cohen-Macaulay module and $\mathfrak{m} H_{\mathfrak{m}}^{0}(M)=0$.

(ii) Every system of parameters of $M$ has the property of parametric decomposition.

Before we give proofs for Theorem 1.1 and its corollary in Section 3, we need some basic facts on good systems of parameters and sequentially Cohen-Macaulay modules, which will be summarized in Section 2. In Section 4 we shall show that the Hilbert-Samuel polynomial of a sequentially Cohen-Macaulay module $M$ with respect to a good parameter ideal can be computed effectively by using the dimension filtration $\mathcal{D}$ of $M$ (Theorem 4.1).

\section{Preliminaries}

Throughout this paper, $R$ is a Noetherian local commutative ring with maximal ideal $\mathfrak{m}$ and $M$ is a finitely generated $R$-module with $\operatorname{dim} M=d$. Let $\underline{x}=x_{1}, \ldots, x_{d}$ be a system of parameters of the module $M$, and we denote by $\mathfrak{q}$ the ideal generated by $x_{1}, \ldots, x_{d}$. For positive integers $n$, we set

$$
\Lambda_{d, n}=\left\{\left(\alpha_{1}, \ldots, \alpha_{d}\right) \in \mathbb{Z}^{d} \mid \alpha_{i} \geqslant 1 \text { for all } 1 \leqslant i \leqslant d \text { and } \sum_{i=1}^{d} \alpha_{i}=d+n-1\right\} .
$$

Let $\mathfrak{q}(\alpha)=\left(x_{1}^{\alpha_{1}}, \ldots, x_{d}^{\alpha_{d}}\right)$ for each $\alpha=\left(\alpha_{1}, \ldots, \alpha_{d}\right) \in \Lambda_{d, n}$. Then $\mathfrak{q}^{n} M \subseteq$ $\bigcap_{\alpha \in \Lambda_{d, n}} \mathfrak{q}(\alpha) M$, and if the equality $\mathfrak{q}^{n} M=\bigcap_{\alpha \in \Lambda_{d, n}} \mathfrak{q}(\alpha) M$ holds true for a system 
of parameters $\underline{x}$ of $M$, we say that $\underline{x}$ has the property of parametric decomposition. Recall that a filtration $\mathcal{D}: \bar{H}_{\mathfrak{m}}^{0}(M)=D_{0} \subset D_{1} \subset \ldots \subset D_{t}=M$ of submodules of $M$ is said to be a dimension filtration if $D_{i-1}$ is the largest submodule of $D_{i}$ with $\operatorname{dim} D_{i-1}<\operatorname{dim} D_{i}$ for all $i=t, t-1, \ldots, 1$, and a system of parameters $\underline{x}=x_{1}, \ldots, x_{d}$ of $M$ is called a good system of parameters of $M$ if $D_{i} \cap\left(x_{d_{i}+1}, \ldots, x_{d}\right) M=0$ for all $0 \leq i \leq t-1$, where $d_{i}=\operatorname{dim} D_{i}$.

Now, let us briefly give some facts on the dimension filtration and good systems of parameters (see $[\mathrm{CC},[\mathrm{CN}]$ ). Because of the Noetherian property of $M$, the dimension filtration of $M$ exists uniquely. Therefore, in the sequel we always denote by

$$
\mathcal{D}: \mathrm{H}_{\mathfrak{m}}^{0}(M)=D_{0} \subset D_{1} \subset \ldots \subset D_{t}=M
$$

with $\operatorname{dim} D_{i}=d_{i}$ the dimension filtration of $M$. In this case, we also say that the dimension filtration $\mathcal{D}$ of $M$ has length $t$. Moreover, let $\bigcap_{\mathfrak{p} \in \operatorname{Ass} M} N(\mathfrak{p})=0$ be a reduced primary decomposition of 0 of $M$; then $D_{i}=\bigcap_{\operatorname{dim}(R / \mathfrak{p}) \geqslant d_{i+1}} N(\mathfrak{p})$. Put $N_{i}=\bigcap_{\operatorname{dim}(R / \mathfrak{p}) \leqslant d_{i}} N(\mathfrak{p})$. Therefore $D_{i} \cap N_{i}=0$ and $\operatorname{dim}\left(M / N_{i}\right)=d_{i}$. By the Prime Avoidance there exists a system of parameters $\underline{x}=\left(x_{1}, \ldots, x_{d}\right)$ such that $x_{d_{i}+1}, \ldots, x_{d} \in$ Ann $\left(M / N_{i}\right)$. It follows that $D_{i} \cap\left(x_{d_{i}+1}, \ldots, x_{d}\right) M \subseteq N_{i} \cap D_{i}=0$ for all $0 \leq i \leq t-1$. Thus $\underline{x}=x_{1}, \ldots, x_{d}$ is a good system of parameters of $M$, and therefore the set of good systems of parameters of $M$ is non-empty. Let $\underline{x}=$ $x_{1}, \ldots, x_{d}$ be a good system of parameters of $M$. It easy to see that $x_{1}, \ldots, x_{d_{i}}$ is a good system of parameters of $D_{i}$ and $x_{1}^{n_{1}}, \ldots, x_{d}^{n_{d}}$ is a good system of parameters of $M$ for any $d$-tuple of positive integers $n_{1}, \ldots, n_{d}$.

Lemma 2.1. Let $\underline{x}=x_{1}, \ldots, x_{d}$ be a good system of parameters of $M$. Then $D_{i}=0:_{M} x_{j}$ for all $d_{i}<j \leq d_{i+1}$ and $0 \leq i \leq t-1$, and therefore $0:_{M} x_{i}^{l}=$ $0:_{M} x_{i}$ for all $l \geq 1$.

Proof. Since $D_{i} \cap\left(x_{d_{i}+1}, \ldots, x_{d}\right) M=0$, we have $D_{i} \subseteq 0:_{M} x_{j}$ for all $j \geqslant d_{i}$. Thus it suffices to prove that $0:_{M} x_{j} \subseteq D_{j}$ for every $d_{i}<j \leqslant d_{i+1}$. Assume that $0:_{M} x_{j} \nsubseteq D_{i}$. Let $s$ be the largest integer such that $0:_{M} x_{j} \nsubseteq D_{s-1}$. Then $t \geqslant s>i$ and $0:_{M} x_{j}=0:_{D_{s}} x_{j}$. Since $d_{s} \geqslant d_{i+1} \geqslant j, x_{j}$ is a parameter element of $D_{s}$ and $\operatorname{dim}\left(0:_{M} x_{j}\right)<d_{s}$. Hence $0:_{M} x_{j} \subseteq D_{s-1}$ by the maximality of $D_{s-1}$. This contradicts the choice of $s$. Therefore $0:_{M} x_{j}=D_{i}$.

Recall that $M$ is said to be a sequentially Cohen-Macaulay module if each quotient $D_{i} / D_{i-1}$ in the dimension filtration of $M$ is Cohen-Macaulay. Notice that the notion of sequentially Cohen-Macaulay modules was introduced by Stanley [St] for the graded case, and it was studied for the local case in [Sch], CN]. Also notice that a special type of sequentially Cohen-Macaulay rings called approximately CohenMacaulay rings was studied very early by Goto G].

\section{Proof of Theorem 1.1}

The following result is an immediate consequence of the definition of a good system of parameters in a sequentially Cohen-Macaulay module.

Lemma 3.1. Let $\underline{x}=x_{1}, \ldots, x_{d}$ be a good system of parameters of a sequentially Cohen-Macaulay module $M$ and $\mathfrak{q}=\left(x_{1}, \ldots, x_{d}\right)$. Then

$$
\mathfrak{q}^{n} M \cap D_{i}=\mathfrak{q}^{n} D_{i}
$$

for all $n \geq 1$ and $0 \leq i \leq t-1$. 
Proof. Since $D_{i+1} / D_{i}$ is a Cohen-Macaulay module with $\operatorname{dim} D_{i+1} / D_{i}=d_{i+1}>$ $d_{i}=\operatorname{dim} D_{i}$ and $\mathfrak{q} D_{i+1}=\left(x_{1}, \ldots, x_{d_{i+1}}\right) D_{i+1}$ for $0 \leq i \leq t-1$, it follows from well-known facts in commutative algebra that

$$
\mathfrak{q}^{n} D_{i+1} \cap D_{i}=\mathfrak{q}^{n} D_{i}
$$

Therefore

$$
\begin{aligned}
\mathfrak{q}^{n} M \cap D_{i} & =\left(\mathfrak{q}^{n} D_{t} \cap D_{t-1}\right) \cap D_{i}=\mathfrak{q}^{n} D_{t-1} \cap D_{i} \\
\ldots & =\mathfrak{q}^{n} D_{i+1} \cap D_{i}=\mathfrak{q}^{n} D_{i}
\end{aligned}
$$

for all $n \geq 1$ and $0 \leq i \leq t-1$ as required.

Let $s$ be a positive integer and $y_{1}, \ldots, y_{s} \in \mathfrak{m}$. For each $1 \leqslant i \leqslant s$ and $\alpha=$ $\left(\alpha_{1}, \ldots, \alpha_{i}\right) \in \Lambda_{i, n}$, we set $Q_{i}=\left(y_{1}, \ldots, y_{i}\right) R, Q=\left(y_{1}, \ldots, y_{s}\right) R$ and $Q_{i}(\alpha)=$ $\left(y_{1}^{\alpha_{1}}, \ldots, y_{i}^{\alpha_{i}}\right), Q(\alpha)=\left(y_{1}^{\alpha_{1}}, \ldots, y_{s}^{\alpha_{s}}\right)$. The following result is due to Heinzer-RatliffShah [HRS, Theorem 2.4]. But we give here the module version of this result proved by Goto-Shimoda [GS2, Lemma 2.1].

Lemma 3.2. Let $s$ be a positive integer and $y_{1}, \ldots, y_{s}$ an $M$-regular sequence. Then

$$
Q^{n} M=\bigcap_{\alpha \in \Lambda_{s, n}} Q(\alpha) M
$$

for all $n \geqslant 1$.

Because the techniques and methods of proof of Theorem 1.1 heavily depend on the works of Goto-Shimoda GS1 and GS2, let us summarize the auxiliary results in GS1] into the following.

Lemma 3.3. With the notation as above the following assertions hold true:

(i) Let $y \in R$ and assume that $0:_{M} y^{\ell} \subseteq y M$ for all $\ell \geqslant 1$. Then $y$ is a non-zerodivisor on $M$.

(ii) Suppose that $Q^{n} M=\bigcap_{\alpha \in \Lambda_{s, n}} Q(\alpha) M$ for all $n \geqslant 1$. Then

$$
Q_{s-1} M: y_{s}^{\ell} \subseteq Q M+\left(0:_{M} y_{s}^{\ell}\right)
$$

for all $\ell \geqslant 1$.

(iii) Suppose that $Q^{n} M=\bigcap_{\alpha \in \Lambda_{s, n}} Q(\alpha) M$ for all $n \geqslant 1$. Then

$$
Q_{i}^{n} M=\bigcap_{\alpha \in \Lambda_{i, n}} Q_{i}(\alpha) M
$$

for all $1 \leqslant i \leqslant s$ and $\ell \geqslant 1$.

In the above lemma, the key is assertion (ii), which is given in the proof of Lemma 3.2 in GS1. By this lemma one gets the following.

Lemma 3.4. Suppose that (1) $Q^{n} M=\bigcap_{\alpha \in \Lambda_{s, n}} Q(\alpha) M$ for all $n \geq 1$ and that (2) $0:_{M} y_{i}^{\ell}=0:_{M} y_{i}$ for all $1 \leq i \leq s$ and $\ell \geq 1$. Then for all integers $1 \leq i \leq j \leq s$, the element $y_{j}$ is a non-zerodivisor on $M /\left[Q_{i-1} M+\left(0:_{M} y_{j}\right)\right]$, so that one has the equality

$$
Q_{i-1} M: y_{j}^{2}=Q_{i-1} M+\left(0:_{M} y_{j}\right) .
$$


Proof. Since conditions (1) and (2) are independent of the order of $y_{1}, \ldots, y_{s}$, we may assume that $i=j$. Then we have

$$
Q_{i-1} M: y_{i}^{\ell} \subseteq Q_{i} M+\left(0:_{M} y_{i}\right)
$$

for all $\ell \geqslant 1$, thanks to (ii) and (iii) of Lemma 3.3. Let $L=M /\left[Q_{i-1} M+0:_{M} y_{i}\right]$ and let $\alpha \in M$ be such that $y_{i}^{\ell} \bar{\alpha}=0$ in $L$ with $\ell \geqslant 1$, where $\bar{\alpha}$ denotes the image of $\alpha$ in $L$. Then $y_{i}^{\ell+1} \alpha \in Q_{i-1} M$, so that $\alpha \in Q_{i-1} M: y_{i}^{\ell+1} \subseteq Q_{i} M+\left(0:_{M} y_{i}\right)$. Hence $\bar{\alpha} \in y_{i} L$, which shows, by (i) of Lemma 3.3, that $y_{i}$ is a non-zerodivisor on $L$. Then the second conclusion is now clear.

Now we are able to prove Theorem 1.1.

Proof of Theorem 1.1. (i) $\Rightarrow$ (ii). Let $\underline{x}=x_{1}, \ldots, x_{d}$ be a good system of parameters of $M$ and $\mathfrak{q}=\left(x_{1}, \ldots, x_{d}\right)$. We prove by induction on the length $t$ of the dimension filtration $\mathcal{D}$ of $M$ that $\underline{x}$ has the property of parametric decomposition. The case $t=$ 0 is trivial. Let $t \geq 1$. Set $\bar{M}=M / D_{t-1}$. Since $\bar{M}$ is a Cohen-Macaulay module, the sequence $x_{1}, \ldots, x_{d}$ is $\bar{M}$-regular. Then $\mathfrak{q}^{n} \bar{M}=\bigcap_{\alpha \in \Lambda_{d, n}} \mathfrak{q}(\alpha) \bar{M}$ by Lemma 3.2. Therefore $\bigcap_{\alpha \in \Lambda_{d, n}} \mathfrak{q}(\alpha) M \subseteq \mathfrak{q}^{n} M+D_{t-1}$. Since $x_{1}^{\alpha_{1}}, \ldots, x_{d}^{\alpha_{d}}$ is a good system of parameters of $M$ for each $\alpha \in \Lambda_{d, n}$, it follows from Lemma 3.1 that $\mathfrak{q}(\alpha) M \cap D_{t-1}=$ $\left(x_{1}^{\alpha_{1}}, x_{2}^{\alpha_{2}}, \ldots, x_{d_{t-1}}^{\alpha_{d_{t-1}}}\right) D_{t-1}$. Now, applying the inductive hypothesis on $D_{t-1}$ we can show with the same method that was used in the proof of Proposition 2.2 of GS2] that $\bigcap_{\alpha \in \Lambda_{d, n}} \mathfrak{q}(\alpha) M=\mathfrak{q}^{n} M$.

(ii) $\Rightarrow$ (iii) is obvious.

(iii) $\Rightarrow(\mathrm{i})$. Let $\underline{x}=x_{1}, \ldots, x_{d}$ be a good system of parameters of $M$ having the property of parametric decomposition and $\mathfrak{q}=\left(x_{1}, \ldots, x_{d}\right)$. Since $0:_{M} x_{i}^{l}=$ $0:_{M} x_{i}$ for all $l \geq 1$ and $1 \leq i \leq d$ by Lemma 2.1. we get by Lemma 3.4 that $\mathfrak{q}_{i} M: x_{j}^{2}=\mathfrak{q}_{i} M+\left(0:_{M} x_{j}\right)$ for all $1 \leq i \leq j \leq d$. Therefore the implication follows by Theorem 3.9 of [CC], and the proof of Theorem 1.1 is complete.

Proof of Corollary 1.2. (i) $\Rightarrow$ (ii). It is easy to see from the hypothesis that $M$ is a sequentially Cohen-Macaulay module with the dimension filtration $\mathcal{D}: H_{\mathfrak{m}}^{0}(M) \subset$ $M$. Moreover, by Lemma 3.1 we have

$$
\left(x_{1}, \ldots, x_{d}\right) M \cap H_{\mathfrak{m}}^{0}(M)=\left(x_{1}, \ldots, x_{d}\right) H_{\mathfrak{m}}^{0}(M) \subseteq \mathfrak{m} H_{\mathfrak{m}}^{0}(M)=0
$$

for any system of parameters $x_{1}, \ldots, x_{d}$ of $M$. This means that every system of parameters of $M$ is good; therefore it has the property of parametric decomposition by Theorem 1.1.

(ii) $\Rightarrow$ (i). First, it follows by Theorem 1.1 that $M$ is sequentially Cohen-Macaulay. Remember by the definition of the dimension filtration of $M$ that $D_{0}=H_{\mathfrak{m}}^{0}(M)$ and $\operatorname{dim} D_{i}>0$ for all $i>0$. Therefore the implication is proved if we can show that $\mathfrak{m} D_{t-1}=0$. Suppose the contrary. Then there is an element $x_{1} \in \mathfrak{m}$ so that $x_{1} D_{t-1} \neq 0$ and $\operatorname{dim} M / x_{1} M=d-1$. Since $d \geqslant 2$, we can choose $x_{2} \in \mathfrak{m}$ such that $x_{2} D_{t-1}=0$ and $\operatorname{dim} M /\left(x_{1}, x_{2}\right) M=d-2$. We observe that the sequence $x_{1}, x_{2}$ and $x_{1}, x_{1}+x_{2}$ are part of systems of parameters of $M$. Therefore

$$
\left(x_{1}^{2}, x_{1}+x_{2}\right) M \cap\left(x_{1},\left(x_{1}+x_{2}\right)^{2}\right) M=\left(x_{1}, x_{2}\right)^{2} M=\left(x_{1}^{2}, x_{2}\right) M \cap\left(x_{1}, x_{2}^{2}\right) M .
$$


Since $M / D_{t-1}$ is Cohen-Macaulay, it follows from Lemma 3.1 that

$$
\begin{aligned}
x_{1} D_{t-1} & =\left(x_{1}^{2}, x_{1}+x_{2}\right) D_{t-1} \cap\left(x_{1},\left(x_{1}+x_{2}\right)^{2}\right) D_{t-1} \\
& =\left(x_{1}^{2}, x_{2}\right) D_{t-1} \cap\left(x_{1}, x_{2}^{2}\right) D_{t-1}=x_{1}^{2} D_{t-1} .
\end{aligned}
$$

Thus $x_{1} D_{t-1}=0$ by Nakayama's lemma, which is impossible. Hence $\mathfrak{m} D_{t-1}=$ 0 .

\section{Hilbert-Samuel polynomials}

A parameter ideal $\mathfrak{q}$ is called a good parameter ideal if it is generated by a good system of parameters. Then, in this section we shall show that for a sequentially Cohen-Macaulay $M$ the Hilbert-Samuel function $H_{\mathfrak{q}, M}(n)=\ell\left(M / \mathfrak{q}^{n+1} M\right)$ has a special expression with non-negative coefficients, which can be computed by the dimension filtration, and this function coincides with the Hilbert-Samuel polynomial $P_{\mathfrak{q}, M}(n)$ for any good parameter ideal $\mathfrak{q}$ of $M$ and all $n \geqslant 1$. Moreover, the sequentially Cohen-Macaulayness of $M$ can be characterized by this expression of the Hilbert-Samuel function.

Theorem 4.1. Let $\mathcal{D}: D_{0} \subset D_{1} \subset \ldots \subset D_{t}=M$ be the dimension filtration of $M$ and set $\mathcal{D}_{i}=D_{i} / D_{i-1}$ for all $1 \leq i \leq t, \mathcal{D}_{0}=D_{0}$. Then the following statements are equivalent:

(i) $M$ is a sequentially Cohen-Macaulay module.

(ii) For any good parameter ideal $\mathfrak{q}$ of $M$, it holds that

$$
\ell\left(M / \mathfrak{q}^{n+1} M\right)=\sum_{i=0}^{t}\left(\begin{array}{c}
n+d_{i} \\
d_{i}
\end{array}\right) \ell\left(\mathcal{D}_{i} / \mathfrak{q} \mathcal{D}_{i}\right)
$$

for all $n \geqslant 0$.

(iii) There exists a good parameter ideal $\mathfrak{q}$ of $M$ such that

$$
\ell\left(M / \mathfrak{q}^{n+1} M\right)=\sum_{i=0}^{t}\left(\begin{array}{c}
n+d_{i} \\
d_{i}
\end{array}\right) \ell\left(\mathcal{D}_{i} / \mathfrak{q} \mathcal{D}_{i}\right)
$$

for all $n \geqslant 0$.

Proof. (i) $\Rightarrow$ (ii). We argue by the induction on the length $t$ of the dimension filtration $\mathcal{D}$ of $M$. The case $t=0$ is obvious. Assume that $t>0$. By virtue of Lemma 3.1. we have a short exact sequence

$$
0 \rightarrow D_{t-1} / \mathfrak{q}^{n+1} D_{t-1} \rightarrow M / \mathfrak{q}^{n+1} M \rightarrow M / \mathfrak{q}^{n+1} M+D_{t-1} \rightarrow 0 .
$$

Therefore, we have $\ell\left(M / \mathfrak{q}^{n+1} M\right)=\ell\left(D_{t-1} / \mathfrak{q}^{n+1} D_{t-1}\right)+\ell\left(\mathcal{D}_{t} / \mathfrak{q}^{n+1} \mathcal{D}_{t}\right)$. Since $D_{t-1}$ is a sequentially Cohen-Macaulay module and its dimension filtration is of length $t-1$, it follows from the inductive hypothesis that

$$
\ell\left(D_{t-1} / \mathfrak{q}^{n+1} D_{t-1}\right)=\sum_{i=0}^{t-1}\left(\begin{array}{c}
n+d_{i} \\
d_{i}
\end{array}\right) \ell\left(\mathcal{D}_{i} / \mathfrak{q} \mathcal{D}_{i}\right) .
$$

Notice that $\mathcal{D}_{t}$ is Cohen-Macaulay of dimension $d=d_{t}$, so we have

$$
\ell\left(\mathcal{D}_{t} / \mathfrak{q}^{n+1} \mathcal{D}_{t}\right)=\left(\begin{array}{c}
n+d \\
d
\end{array}\right) \ell\left(\mathcal{D}_{t} / \mathfrak{q} \mathcal{D}_{t}\right)
$$


Hence

$$
\ell\left(M / \mathfrak{q}^{n+1} M\right)=\sum_{i=0}^{t}\left(\begin{array}{c}
n+d_{i} \\
d_{i}
\end{array}\right) \ell\left(\mathcal{D}_{i} / \mathfrak{q} \mathcal{D}_{i}\right)
$$

for all $n \geqslant 0$.

(ii) $\Rightarrow$ (iii) is trivial.

(iii) $\Rightarrow(\mathrm{i})$. Since the following sequence is exact:

$$
D_{t-1} / \mathfrak{q}^{n+1} D_{t-1} \rightarrow M / \mathfrak{q}^{n+1} M \rightarrow M / \mathfrak{q}^{n+1} M+D_{t-1} \rightarrow 0,
$$

we get $\ell\left(M / \mathfrak{q}^{n+1} M\right) \leqslant \ell\left(D_{t-1} / \mathfrak{q}^{n+1} D_{t-1}\right)+\ell\left(\mathcal{D}_{t} / \mathfrak{q}^{n+1} \mathcal{D}_{t}\right)$. Therefore, by induction on the length of the dimension filtration we can show that

$$
\ell\left(M / \mathfrak{q}^{n+1} M\right) \leqslant \sum_{i=0}^{t} \ell\left(\mathcal{D}_{i} / \mathfrak{q}^{n+1} \mathcal{D}_{i}\right)
$$

On the other hand, since

$$
\ell\left(\mathcal{D}_{i} / \mathfrak{q}^{n+1} \mathcal{D}_{i}\right) \leqslant\left(\begin{array}{c}
n+d_{i} \\
d_{i}
\end{array}\right) \ell\left(\mathcal{D}_{i} / \mathfrak{q} \mathcal{D}_{i}\right)
$$

for all $0 \leq i \leq t$, it follows from the hypothesis that

$$
\ell\left(M / \mathfrak{q}^{n+1} M\right)=\sum_{i=0}^{t} \ell\left(\mathcal{D}_{i} / \mathfrak{q}^{n+1} \mathcal{D}_{i}\right)=\sum_{i=0}^{t}\left(\begin{array}{c}
n+d_{i} \\
d_{i}
\end{array}\right) \ell\left(\mathcal{D}_{i} / \mathfrak{q} \mathcal{D}_{i}\right)
$$

Therefore $\ell\left(\mathcal{D}_{i} / \mathfrak{q}^{n+1} \mathcal{D}_{i}\right)=\left(\begin{array}{c}n+d_{i} \\ d_{i}\end{array}\right) \ell\left(\mathcal{D}_{i} / \mathfrak{q} \mathcal{D}_{i}\right)$ for all $n \geq 0$ and $0 \leq i \leq t$. Thus $\mathcal{D}_{i}$ is Cohen-Macaulay for all $0 \leq i \leq t$, and this completes the proof.

\section{ACKNOWLEDGMENT}

The authors would like to thank the referee for useful suggestions. Especially, Lemma 3.4 stated by the referee reduces remarkably the proof of Theorem 1.1.

\section{REFERENCES}

[CC $]$ N. T. Cuong and D. T. Cuong, On sequentially Cohen-Macaulay modules, Kodai Math. J., 30 (2007), 409-428.

[CN] N. T. Cuong and L. T. Nhan, Pseudo Cohen-Macaulay and pseudo generalized CohenMacaulay modules, J. Algebra, 267 (2003), 156-177. MR1993472 (2004f:13012)

[G] S. Goto, Approximately Cohen-Macaulay rings, J. Algebra, 76, No. 1 (1982), 214-225. MR 659220 (84h:13033)

[GS1] S. Goto and Y. Shimoda, Parametric decomposition of powers of ideals versus regularity of sequences, Proc. AMS, 132, No. 4 (2004), 929-933. MR2045406 (2004m:13008)

[GS2] S. Goto and Y. Shimoda, On the parametric decomposition of powers of parameter ideals in a Noetherian local ring, Tokyo J. Math., 27, No. 1 (2004), 125-135. MR2060079 (2005d:13005)

[HRS] W. Heinzer, L. J. Ratliff and K. Shah, Parametric decomposition of monomial ideals. I, Houston J. Math., 21 (1995), 29-52. MR1331242 (96c:13002) 
[Sch] P. Schenzel, On the dimension filtration and Cohen-Macaulay filtered modules, in Proc. of the Ferrara meeting in honour of Mario Fiorentini, University of Antwerp Wilrijk, Belgium (1998), Dekker, New York, 1999, pp. 245-264. MR1702109(2000i:13012)

[St] R. P. Stanley, Combinatorics and Commutative Algebra, Second edition, Birkhäuser Boston, 1996. MR1453579 (98h:05001)

Institute of Mathematics, 18 Hoang Quoc Viet Road, 10307 Hanoi, Viet Nam

E-mail address: ntcuong@math.ac.vn

Institute of Mathematics, 18 Hoang Quoc Viet Road, 10307 Hanoi, Viet Nam

E-mail address: hltruong@math.ac.vn 